\title{
EDITORIAL
}

\section{New horizons in gastroenterology: infection, inflammation, and immunity}

\section{J G Farthing}

Correspondence to: Prof. M Farthing, Faculty of Medicine, University of Glasgow, 12 South Park Terrace, Glasgow G12 8LG, UK:

m.farthing@clinmed.gla.ac.uk .....................

Gut 2002;50(Suppl III):iii I

G astrointestinal diseases continue to have a major morbidity worldwide. Intestinal infections are the most common disorders of the gastrointestinal tract and infectious diarrhoeal disease continues to be a major cause of morbidity and mortality. Almost 5 million preschool children still die each year from infectious diarrhoea, either from dehydration and acidosis due to fluid losses or because of the direct effects of invasive micro-organisms on gut structure and function. New micro-organisms have emerged during the past 20 years of which Helicobacter pylori has had the most profound impact on our understanding of the pathogenesis of disease, notably upper gut disorders such as gastritis, peptic ulceration, and gastric neoplasia.

Inflammation following intestinal infection is usually appropriate to the stimulus and generally resolves once the infection has been cleared. However, in non-specific inflammatory bowel disease, inflammation continues in the absence of an obvious inflammatory stimulus. There is now compelling evidence that the normal bowel flora is an important trigger to chronic intestinal inflammation. In addition, there is increasing evidence that the genetic background of the host plays a dominant role in determining the type and chronicity of disease expression. Despite frustratingly slow progress in the development of our ideas on pathogenesis of non-specific inflammatory bowel disease, this has not prevented the evolution of new therapies. These include the new steroids and new salicylates, small therapeutic molecules aimed specifically at critical targets in the inflammatory cascade, and probiotics.

This supplement to Gut contains contributions from two scientific meetings in 2001 sponsored by the European Institute of Health Care. It was a privilege to plan and chair these meetings with my colleague Francesco Pallone and to put together this supplement. We devised the scientific programme independently without any constraints or advice from our sponsor AstraZeneca. Although the topics exhibit some diversity, important unifying themes in infection, inflammation, and immunity in the gut do clearly emerge. The internationally renowned faculty has provided authoritative chapters, which at least in 2001-02, are state of the art. 